\title{
SUPPLEMENTARY CATALOGUE
}

*OF

\section{RARE AND NEW FRUITS,}

\section{IN ADDITIION TO THOSE COMPRISED}

IN THE 34TH EDITION OF

THE GENERAL CATALOGUE OH FRUIT TREES, \&

CULTIVATED AT

PRINCE'B LINNEAN BOTANIC GARDEN AND NURSERIES, •

FLUSHING, NEAR NEW-TORK.

\section{Wm. 13. Prince \& Co., Proprietors.}

Nore. - As the accessions to our collection of all highly estimable varietic. of Fruits, engages our constant attention, it necessarily arises that we aro ir pnssession of them some time before we can announce them in the regular pditions of our General Catalogue. Amateurs may therefore confidently rels, that whenever any very estimable varieties are announced in Europe, or in nur own conntry, that we are among the very first to possess them, and as wh propagate them rapidly, we can always supply them as soon or sooner than whers, and at reasonable rates.

The newest varieties of the following Fruits, are but one year grow from the graft or inoculation-the others are of two tears growth.

* The Asterish denotes Fruits of American origin.

\section{APPLES - 25 To 37 CENTS ЕACH,}

EXCEPT WHERE OTHERWISE NOTED.

* Beard Burden.

Belle du Havre.

* Broadwell.

Western Romanite.

* Burnham Greening.

Coe's Golden Drop.

* Cooper, (of Uhio)

*Corsonite.

Crab, Spectabilis Riversi, $50 \mathrm{cts}$.

-, Weeping, $50 \mathrm{cts}$.

*Bough, Tart

* Detroit, White

- Detroit, Red

* Early Joe.

Early Red Margaret,

Eng'ish Red Juncating.

Borden's Early.

* Easly Piper.

Early Summer Pippin, Ronald's, 50 cts.

English Ruscet.

- Estin.

Aromatic Russt.

- Fall Ginnetting.

- French's Sweet.

- Guarden Striped.

- Genesee Pippia.
*Gillet's Red, (of Ohio.)

Hambledon Deur-ans, $50 \mathrm{cts}$

*Heath's Early Nonsuch.

*Hyde's Large Red.

* Indian Rareripe.

- Lady Healy's Nonsuch.

* Large Early Yellow, (Ohio.)

*Lindley's Nonpareil, (N.C.) no su.

*Lincoln.

* Long Island Wine, 50 cts.

* Lyman's Pumpkin Sweet.

* Maclean's Favorite.

* Martin.

- Margold, American

* Maryland Lady's Blush.

* Masters' Red Winter.

* Mathews' Stripe.

* May.

\section{* Melon.}

* Mohawk Pippin, (Spinuer's, )

Monstrueux, $50 \mathrm{cts}$.

* Mother.

* Mouse.

* Nottingham Browa.

- Paradise, Winter Swase

* Peachpond Sweet.

\section{1}


Pearmain, Claygate, $50 \mathrm{cts}$.

English Sweeting, of R.I.

Pippin, Holland

*_, Cranberry

- Lemon, (Ohio)

-_, Nyack

*___ Sprisghill, (6 trees for \$5.)

Sirong's Newtuwn Pippin.

- Sturmer

Pomme royale, or Pound Royal.

*Power's Large Siberian Crab.

* Princeall.

* Randolph's Cluster.

* Red Canada.

* Reinette, American Golden, 50 cts.

- White Spanish

Reinette blanche d'Espagne.

Roth Kruger.
Russet, Pineapple, $50 \mathrm{cts}$.

*..._, Pumpkin

Sweet Russet.

* Shepherd's, (Ohio)

* Spitzemberg, Spring Hill, (6 trees for $\$ 3$, )

* Sterling Beauty.

*Stump.

* Sweeting, Wells'

English

Ramsdell's Red Pumpkin Sweeting.

*Victuals and Drink.

-Twenty ounce. Cayuga Red Streak.

*Waxer, or Belmont. Gate-White apple.

*Williams' Carolina, 50 cts.

Wilmot's Early Seedling, $50 \mathrm{cts}$

* York Greening.

\section{PEARS. - E^}

Ah mon Dieu.

Arbre courbé.

-Areson's Doyenné.

Archduke Charles.

Balue.

Belle de Feron.

- de Moisé.

- de Troyes.

- excellente.

Heloise.

Bergamot, March , Truckhill

Bergamotte de Parthenay.

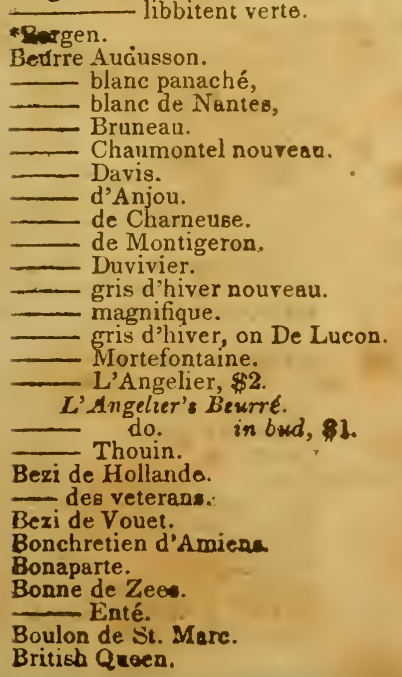

Caillot rosat, English

Calebasse de Nerkman.

*Calhoun.

Captif de St. Helene

Chapman's Early.

- Maria.

Chaumontel Belge.

*Citron.

Colmar Charnay.

Comtesse de Lunay.

*Diallas.

De Juvardeil.

De Pape, on de St. Pierro.

Delice de la Cour.

Dingler.

Doyenne d'Alençon.

- musqué.

—_ d'hiver nouveas.

— d'eté, (true)

- gris d'hiver nourean.

_ gris jaune d'h

Duchesse de Mars. d'Orleans.

Duc de Bordeaux.

* Edwards.

Epine Dumar.

d'été.

Lpiscopal.

Esperine.

Ezinowesser.

Ferdinand Meester.

Figue verte.

d'Alengon.

Fontarablo.

Garnier.

Girardon.

Girofle.

Gratioli, (of Jersey)

Josephine de Malio

Helicte Dundas.

- Heary. 
* Henrietta.

*Hill's Fall Butter, (of Ohio)

*Hull.

*Imperial Sugar, (N. Carolina)

Inconnue Van Mons.

Las Casas.

Leon le Clerc of Van Mons.

*Locke.

Louise de Prusse.

Luquet d'Allemagne.

Arabille.

Marie Louise Delcour.

Mes Delices.

* IIoore's Pound.

Neill, Van Mons

* Oliver's Russet.

Orange d'été.

* Osburn, (Ohio)

* ()ustremall.

l'arfuin d'hiver.

Poire Belle Henriette.

Puire de M. Bonnet.

St. Dorothée.

- Rimniri....

Shilippe, or Double Philippe

Plomgastel.

\section{Deasnas.}

\section{Dumas.}

*Polk.

Pratt.

*Queen of August, large, ouperior, extra, $\$ 1$.

Rahut, or Deus fois l'an.

Reine d'hiver.

Ridelle.

Rousselet de Stoutgard. perdreau.

St. Germain nouveau.

St. Marc.

Soldat laboureur.

Souveraine d'été

*Stansel's Newton.

St. Francois.

St. Joseph.

St. Michael d'hiver.

* Summer Virgalieu.

Surpasse Madeleine.

Tarquin des Pyrenées.

Tavernier de Boulogne.

*Tyson.

Van Mons of Leon le Clame Van Mons Leon le Clerc.

Vicomte de Spoelberch.

Vilaine de St. Florent.

Wellington, or Waterloo.

Wilhelmina.

William.

Pears on Quince for Dwarfs or of Quenouille form can be supplied of above 100 fine varieties.

CHERRIES.-EACH 50 cENTS.

J3auman's May.

Bigarreau de Mai.

Belle de sceaux, $\$ 1$.

*Buwne's Clarion, \$1.

Buttner's Black Heart.

Morello.

Cerise do Ravaen.

* Columbia, $75 \mathrm{cts}$.

*Coe's Transparent, \$1.

Downing's Red Cheek,

Early White Heart. (Downing.)

Gold striped leaved, ornamental, $\$ 1$.
Holland Bigarreau. Bigarreau de Hollande.

Imperial Morello.

*Ives' Mottled Seedling.

Lemercier, $75 \mathrm{cts}$.

*Prince's Early Black Heart, very estimable early variety, $75 \mathrm{cts}$.

Reine Hortense, $\mathbf{7 5}$ cts.

Rivers' Early Heart.

Rumsey's Late Morello.

Walsh's No. 1.

No. 2

Werder's karly Black.

\section{PLUMS.-EACH 50 cENTS.}

A pricot Plum of ' $\mathbf{I}$ ours, $\mathbf{7 5} \mathrm{cts}$. Abricotée.

Bradihaw, 75 cts.

Buel's Favorite.

Coutche de Dorel, $\$ 1$.

Chapman's Prince of Wales, 75 cts.

Coxe's Incomparable.

*Dennison's Albany Bequty.

*Dennison's Red.

* Superb.

Domine Dull, (distinct.)

French Long Blue.

Du Kor, \$1.

* Early Lienessee.
English Yellow Gage. Reine Claude blanche.

Fellenberg, 75 cts.

Forbus Gage.

Fonday.

Golden Gage, 75 cts.

Guthrie's Late Green, 75 cts.

- New Apricot Plum, 75 ct.

- Taybank, $\$ 1$.

Green Roman, $\$ 1$.

Verte bonne de Rome.

*Henrietta Gage.

Howland's Yellow.

*Hudson Gage. 
"Ives' Washington Seedling.

King of Plums, 75 cts.

*Langdon's Seedling, $75 \mathrm{cts}$.

* Lawrence's Yellow Egg, 75 cts. Purple Gage, $75 \mathrm{cts}$. Oval Blue.

Merveille de New Kent.

Montfort, 75 cts.

*Mulberry.

* Orange.

Peach Plum (of France.)

Prunes des bois.

*Purple Favorite.

Reine Claude de Bavay, $75 \mathrm{cts}$.
Reine Claude d'Octobre, $75 \mathrm{cts}$

Rivers' Early, No. 2.

*Rockland Great Grage.

Royal Green Gage, 75 cts.

Saint Etienue, 75 cts.

*Thomas.

*Tory, or August Damson.

Violette de Belgique, 75 cts. Belgian Violel.

Virgin, (Thompson.)

*White American Cherry.

$$
\text { Apricot. }
$$

Abruoté blanc.

* Winter Bolmer.

de Toussaint, 75 cts.

\section{PEACHES.-25 CENTS, EXCEPT THOSE NOTED.}

\section{(C. denotes Cling:tones.)}

Acton Scott.

\section{* Baltimore Beauty.}

\section{- Barnaru.}

Belle de Chatenay, $50 \mathrm{cts}$.

*Bough, 50 cts.

* Camiden.

*Congress, C. (genuine)

*Druids Hill.

*Duchess Mignone, $50 \mathrm{cts}$.

* Early Newington Freestone.

* Early Tillotson, (mildews.) White, $50 \mathrm{cts}$.

*Flushing Heath, C. 50 cts.

* Imperial, C. very large, ripe in Oct., 50 cts.

French Violet, $50 \mathrm{cts}$. Apricot Peach, 50 cts.

White Nutmeg, eariicst, 50 cts. * Grant's White October, 50 cts.

Green Catharine, common variely, erroneously so called.

Grosse Admirable jaune, $50 \mathrm{cts}$. - Mignone Incomparable, $50 \mathrm{cts}$.

*Hart's Seedling, $50 \mathrm{cts}$.

*Honest John.

* Jersey Rareripe,

*Kimber's Favorite Yellow.

*Leopold.

* Lignum rubrum, a curiosily.

* Marie Antoinette, (? syn.)

* New Sweetwater, very curly, 50 cts.

*Orange Red.

Parie Monstre, C. $50 \mathrm{cts}$.

\section{NEC'TARINES.-37' To 50 cENTS.}

Prince's Excelsior, ( 6 trees in bud $\$ 5$ )

*___ Fortunatus, C.splendid, 75 cts.

*___ White October, C. very esti-

an ahle. (6 trees for \$3.)

* ".un..., White Uctonc....

trees for $\$ 3$.)

*Pope's.

Royal Pompone, C. monstrous, exce!lent, ripe Oct 20. (6 trees for $\$ 5$.)

Scarlet Admirable, $50 \mathrm{cts}$.

Sulhampstead.

*Scott's Extra Early, 50 cts.

* _- Nonpareil, 50 cts.

* September Red liareripe.

Sernach.

Smith's Magdalen, 50 cts

* - Mammoth, (6 trees for $\$ 5$.

*__ Yellow October, C. 75 cts.

*Spring Hill Orange, 75 cts.

* Strawberry.

* Stump the World. World's Beater.

*Tippecanoe, $\mathrm{C}$.

Vandyke, large, very fine, ripe Aug. 15.

$V$ anguard, (distinct from Nobleese.)

*TVaxen Misnone, 50 cts.

*Washington Cling, (gcnuine.)

*Weeping, ormamental, $37 \mathrm{cts}$.

* Thite Imperial.

* Thitehead's Red Heath, C.

* TViley's Nutmeg, very early, $50 \mathrm{cts}$

*Yard's Favorite, 50 cts.

Broọmfield.

Hardwicke Seedling.

Old White.

\section{APRICO'TS.}

Akarish, (from Persia) $5 \mathrm{cts.}$

Alberge le gros, $50 \mathrm{cts}$

Early Red, 37 cts.

Gros Musch, o
Grog Muscat, 37 cts.

Vert Muscat.

St. Ambroise, $75 \mathrm{cts}$ 
DUTCH MEDLAR.

Aonstreuse, $75 \mathrm{cts}$.

Variegated leaved, $50 \mathrm{cts}$.

\section{GRAPES.}

For an admirable collection of new and very select varieties of the most estimable Table Grapes, see our General Catalogue page 39, and our Supplement Catalogue, No. 8. We also call attention to the 13 new Hybrids from the Isabellas, which are there announced. In addition to the fine collection of Table Grapes, we now offer 50,000 plants of native varieties, suitable for market and for making wine, and the time is not distant when our country, which has been prëeminently marked out by the Deity as the "Land of the Vine," will be dotted over with luxuriant vineyards, which, when planted with our native varieties, will yield crops six-fold as great as the vineyards of Europe.

\section{RASPBERRIES.}

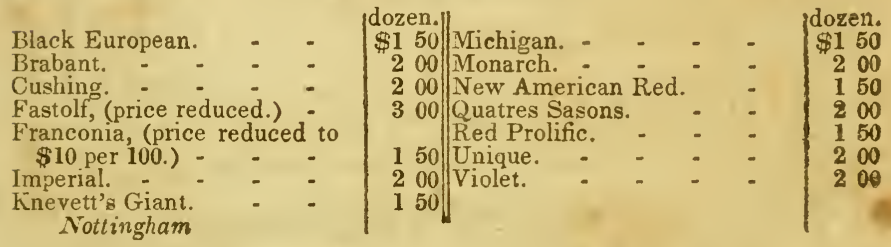

\section{CURRANTS.}

French Large White, $37 \mathrm{cts}$.

Large Red, 37 cts.

Large Red Provence, (\$3 for 6.)

-White do. silver edged leaves, (\$3 for 6.)

N. B. The two preceding varieties are the largest of all Currants, and are very distinct and of remarkably vigorous growth, and are in no other Nursery in England or America.

White Transparent, $37 \mathrm{cts}$.

Blanc iransparent.

Symphoria fuchsoides, ornamental, $50 \mathrm{cts}$.

\section{STRAWBERRIES.}

THs splendid Collection of varieties enumerated in the 34th edition of or General Catalugue have been greatly reduced in prices, and we make it a rule to charge always as low or lower than similar kinds can be obtained elsewhere, to say nothing of the superiority of our Collection in excellence and accuracy over every other. The most of the following varieties have been added to our assortment since our General Catalogue was published. More than 50 other estimable varieties are now in our Collection, which we shall annuunce to the public hereafter when fully proven.

P. denotes pistillate varieties; S. staminate.

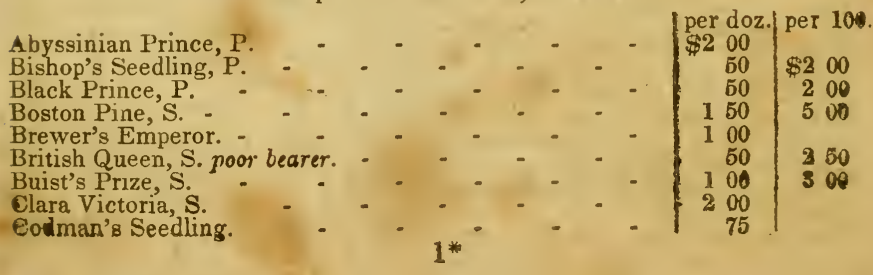


Deptford Pine, $\mathrm{S}$.

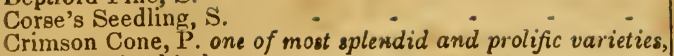

the genuine kind.

Crimsou Pine, S.

Garnestone Scarlet, $\overrightarrow{\text { S. }}$.

French Queer.

Globe Scarlet.

Hovey's Seedling, P.

Hudson's Bay, S. and P.

Hudson, P. (from Cincinnati.)

Iowa, or Prairie, S.

Jenny's Seedling.

Lady's Finger.

Large Farly Scarlet, S.

Lizzie Randolph, P. large, round, proliflc. -

Montevideo Pine, S.

Myatt's Eliza, S. poor bearer.

Necked Pine, (from Cincinnati.)

North's Victory.

Parisian Alpine, S.

Primate, S. large, splendid, deep scarlet, very productive, a seed'ing from Prince Albert.

Primordian, P. the finest and most productive early variety.

President, S.

Prince Albert, S. genuine, large. sp'endid, deep scarlet.

| per doz. per 100 .

$\$ 100 \$ 500$

$100 \quad 3$ of

$100 \quad 500$

$150 \quad 500$

$50 \quad 200$

200

$5 0 \longdiv { 2 0 0 }$

$37 \quad 150$

251100

$50 \quad 200$

$5 0 \longdiv { 2 0 0 }$

75

200

25

200

500

50

75

75

100

100

300

300

300

100

200

300

300

5 on

N. B. The plants sold under this name are almost invariably a spurious and inferior variety.

Princess Alice Maude, $\mathbf{S}$.

Ross' Phoyal, S.

100

150

1000

10 an

Stoddard's Washington Alpine, is the common English Red

500

Wood.

Swanstone, S.

Taylor's Seedling, (Ohio.)

Unique, peculiar fo $m$, sweet, delicate flavor, prolific.

Willey, S. is a syronzm.

The following, with about 25 other varieties, can be supplied at the operne of Spring, or a few plants of each can be supplicd the present Autumn at 1 for each variety :-Adriana, Caleb Cope, Cushing, Emily, Hancock, Mary, $\checkmark$ irginia, William Henry, \&c.

\section{RAPE AND NEW TREES AND SHRUBS.}

morpha canescens, dwarf.

Ash, Silver striped; spiendid.

Bignonia atrosanguinea.

Calycanthus heterophyllus.

Clematis napanifolius.

Glematis napalensis, autumn flowering.

Caraganu haladendron.

- altagana.

Colutea microphylla.

Cratægus Douglassii.

Cytisus austriaca.

Eleagnusi flava, or European Silver Trea.

Elder, Silver dusted

Parsley leaved

Elm, Gold striped

Enonymus pendula. 
Enonymus atrovirens.

- coccuseus, or Scarlet fruited.

Hydrangea Japonica, beauliftal.

Hypericum calycinum.

Magnolia glanca semiplena.

Populus monilifera, Gold striped.

- hudsonica, Gold striped.

_remuluides.

Privet, Box leaved, handsome.

Prumus padus-pendula.

Rhamnus alnifolius.

Ribes sanguimeum atrorubens.

- divaricatum.

- hybridum gordonianum.

Robinia floribunda.

Rubus spectabile.

Spiræa acutifolia.

- grandiflora.

- rotundifolia.

Syringa emodii.

Taxodium sinensis, pendula.

Wistaria alba.

Xylosteum , Pale blue, long spikes of flowers, vigorous growth.

\section{EVERGREEN TREES AND SHRUBS.}

\section{abies morinda.}

- Douglassii.

- Clanbrassiliana.

- Cephalonica.

Araucaria imbricata.

Berberis heterophylla.

Cupressus torulosa.

Cotoneaster buxifolia.

affinis. affinis.
\$1 5c/Juniperus excelsa.

\section{RHODODENDRONS}

Recently received in addition to those enumerated at pages $\mathbf{7 4}$ and 106 of our General Catalogue. We can supply $R$. ponticum and Catawbiense of different sizes, by the dozen or hundred, at reasonable rates.

Catawbiense purpurea. hybrida.

Dauricum atrovirens.

\section{Glomeratum.}

Maximum reseum. Master's Rose-colored, New Silver striped. Vivaticum.

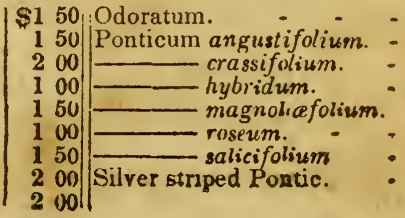




\section{NEW CHRYSANTHEMUMS.}

Those thus noted + are 37 cents-and those not notcd are 50 cents.

tAdventure, dark rose:

+Bathula, mottled rose.

Belle du Rhin.

Bicolor expansa, white, yellow centre, full double, expanded, beautiful.

Comète.

tComtesse Suchette, delicate lilac.

- Lamballe.

Delight.

tDemosthenes, claret, very full and fine.

tDavid, superb yellow, very extra.

†Duchesse de Minthelle, silvery lilac, very neat, elegant.

Duclos, mahogany colored.

Frankee, deep red.

tGrand Duke, fine white, full, cupped.

Limon blanc, white, straw centre.

†Marchioness, fine lilac

+Marie Antoinette, delicate pink blush.

Mécure.

Minerva bicolor, large, very full, delicate lilac, centre sulphur.

tQueen, mottled rose, full, fine.

Rose blanchatre, superb rosy white.

†Sulphurea elegans, beautiful dark yellow, on

†Sultan, white.

PHLOX.-Additional Varieties.

25 CENTS EACH, EXCEPT WHERE OTHERWISE NOTID.

12 varieties for $\$ 4 ; 24$ do. for $\$ 7 ; 50$ do. for $\$ 12$.

Acuminata atrorubens minor. lilacina bella.

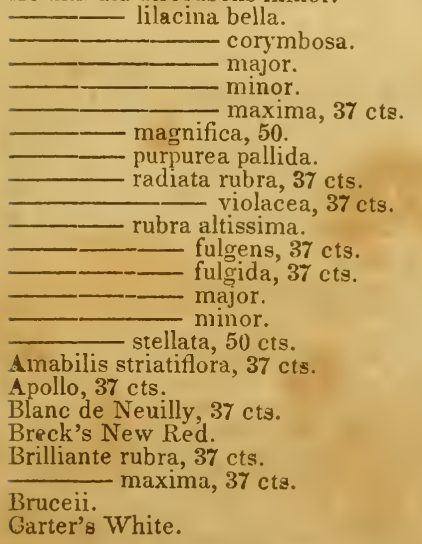

Charles, 37 cts.

Coldryana.

Elegantissima, 37 cts.

Frelinghuysen, $37 \mathrm{cts}$.

Grandiflora, vel Gigantea, $37 \mathrm{ets}$

Harrisonia, white.

Henry Clay, majestic, vigorous habit, beautiful variegated flowers, 50 cts.

Hortensoides, $37 \mathrm{cts}$.

Lawrencea, pure white, 37 cts.

Lilacina grandiflora.

Murrayana.

Mutabilis.

Nymphæa alba, $50 \mathrm{cts}$.

Oeuil de Lynx, 37 cts.

Paniculata nivea, 37 cts.

Princesse Mariame, $37 \mathrm{cts}$.

Reevesii, 37 cts.

Rubra striata, 50 cts.

Sickmannii.

Speciosa, 37 cts.

Van Houtei, $50 \mathrm{ct}$.

Wilderi.

\section{IRIS.-Additional Species.}

25 CENTS EACH, EXCEPT WHERE NOTED.

Adansoni, 50 cts.

Bicolor, splendid yellow with black spots, $\$ 1$.

Bengii, splendid yellow, 50 ets.

Coelestis, $50 \mathrm{cts}$.

Flava. 
riramin.'2.

(ive den st adii.

I Lematophyilic, $3 \pi \mathrm{cts}$.

Lliuclia, $3 \%$ cts.
Maritina.

Nothis, : $37 \mathrm{cta}$.

Stenogyнa, 37 ct $=$.

Vanderbure, $50 \mathrm{cis}$

\section{CARNATIONS AND PIC TEES.}

Ahove 100 new varieties have been imported in fiddition to the splendid coll-ction th ou General Cualngue, pages 97 and 107 -and will be stpplied in

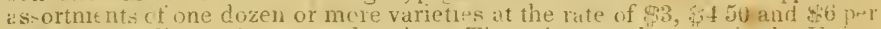
dozn, acco ding to beauty and larity. There is no coliection in the $[$ ?.im that can comprie with the 200 vireities we now olier.

\section{G CANELLIA JAPONICA.}

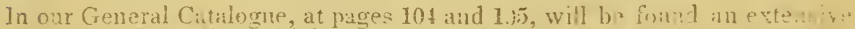

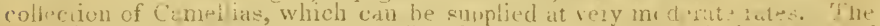

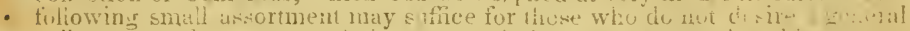

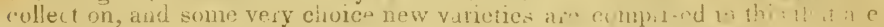

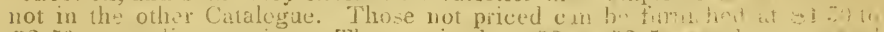

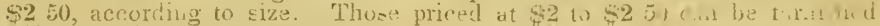
of illcre ised slie at 50 ets. to $\$ 1$ ( $/ 0$ acivalict on those ridtes. Alba plena, or Touls:: White.

\section{Do.}

Lo. hova.

Alba grandiilora.

Alsatica.

Althæifcra.

Amabilis.

Antwer iensia

Atromibena.

Jiarni.

di...ili, or Lecenat smpelua. jiariezina.

bestonia.

Jrucea: a.

('anmbe! ii

Candidisima.

( $i$ : 1 n'a

C'arswelliana.

('landlerii.

('olviliii

('omte cie Neupterg

Conspictid.

Ciqueli.

1 orollata.

\section{("riciuta}

Cummingi.

bark Fii cens.

l)eers.

Delecta.

leticatissima.

l.onkle is

inchese d. Nemours. d Oilmins.

Filegans (Chandler)

binịlie grandiffore.

Eirectit.

fimbn: ra.

I'loyi.

Formosal.

Frankf tien-is. $\mid \begin{aligned} & 43 \\ & 4\end{aligned}$

?

3

2

4

\section{2}

$+$

2

2 5ul Necbracer. i.

ícli j. =ilu t.

2

Oeniclanes.

() $x$ rintenzis.

Oxiyglomima shyora.

8

3

250

Perciveili.

Perf:erion, or ('ipendishi.

Puly tila ine rn 1.2 .

P'Pes': Eic!yp.e.

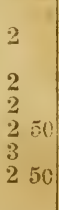

Prese

P’rinc s T J inville.

Pulchprims.

(queen Tictoria.

R ammoncliffora.

fieticinlata.

liosa n?'rludi.

líger-a plani:?

250 sisanyin ro.ea p'ena.

2 "Cherwoodii

25 i Spreffortialla.

25

2 
triumphons. . .

Ufomi. .

Tandesii carnca.

Variegated, or štriped.

Venosid.

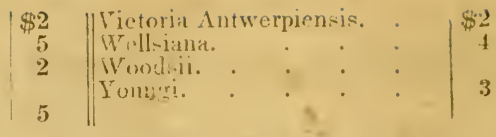

\section{CHINESE AZALEA, or AZALEA INDICA.}

Thre varictios can be s!applied in ardition to those enumsrated at page 7.3 of the ranerul ('italogne, price $50 \mathrm{cts}$. to 462 , according to their rarity and the size of the plants.

dudinennia.

Amabilis:

Aurantiaca.

Candidissima.

Corulea.

'occinea grandiflora.

_ - speijsu.

Crianoiser senutplena.

('muinghami.

Dihlemi.

1) Hotsmina.

Fiats Whito.

$\mathrm{l}+\mathrm{il} \leq \mathrm{u}$.

(ileci-temsii, sis 150 .
Grandintora. Neriiflora. lilacina nova. 1'inichella.

Hesdersonii.

Irne-cens, 5u cts.

linearnata.

Iudica, fol. varieg.

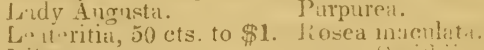

Lilate:

Lilieina.

II.cinthi, 5t) cts.

LI iz'pi'l.

Mizorati.

silinerii
I'ulcherina.

Palchra speciosa.

Puncea.

J'arpurea.

Smithii.

Rubrida.

skrimikersi.

Smilh's Liglit I'ink.

Simperba.

Thomasi.

\section{PELARGONIUMS; cn GEn A}

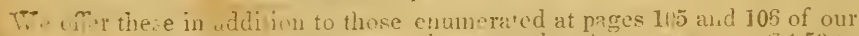

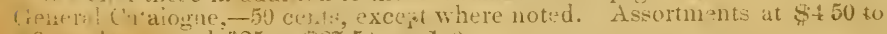

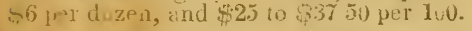

\section{! 'ind}

t'b. asinfore.

A.. n m.t.

Lle.. ithiti.

Arabelis:

6.: :

Pridetroment

( inb idire Belle, \$1.

( आ10Lim, \$1.

( + $1 . \mid 1,1)$

(ind, dism.

('ount de P'aris.

('onli, slation.

('onq

( गу

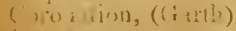

(1) (1) 311 .

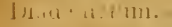

vido.

Dowione Q recu.

Jiclype.

Einin!

(i) 111 in ror, (IIodge)

linth thile s.

Finmy Garth
Iincily.

finterii rosét.

Hebe.

Ifereviles.

Jusiams j)walf.

Jenny Lind, के 1.

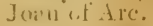

li!b:ı.

isings.

Ning John.

latdy flomero.

- II tyoress.

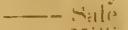

- Tillims.

L, il Jiveren.

1, t). 1 .

Uन่ आ1 I.t-limi.

Alud ü 1 Irivi:

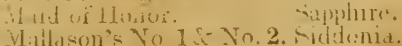

Ovid.

l'arason.

P.ict.

linchel.

livenzi.

liosettil.
I'irfiction, Girth.

P'ëaninent

link l'relfection.

Prilila Dunna.

P'rucess fiophin Itatitelat.

(Luen I'hillippi.

- of Silrint.

(Qnecu's vinpert)

Rival Q queen.

Rnilualite.

\section{II tliil.t. \\ - irir Robert Peel.}

inder). 4 is.

Ires. Lestur.

i) $\cdots(101)$

(i)fit) 11 .

().1Vir I'wist.

(i)inor liovent.
Siyl $\left.l_{1}\right)$.

Lilit

Yictory.

II ildfire.

II lison's Enchantress, \$i

Troudel.

\section{HUCHSIAS.- 0 CENIS, EXCEPT THOSE NOTED.}

Audot, 37 cets.

13alloonia stuperba.

Baveretomii.

lin'alliy.
Bridier.' m

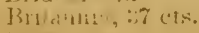

( thitvall 11.

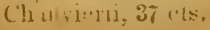

(uccilieal, 25 cts.

('urica, i,7 cts.

(')rymbillura.

Corymbus: 37 ' 12. 
Decora, or Transparens, Globosa, 37 cts. $37 \mathrm{cts}$.

Defiance, $3 \pi \mathrm{cts}$. Hector.

Ivory Gem, or Iveryana,

Duke of Wellington, $37 \mathrm{cts}$. $37 \mathrm{cts}$.

Elata.

Eileas.

Enchantress.

Kentish Hero, 37 cts.

La Chinoise.

Lindleyana.

Eppes Queen of Virgins, \$1 Magnifica.

Eppesil.

Lirecta elegans.

Exoniensis.

Florence, $37 \mathrm{cts}$.

Fulgens, 37 cts.

Gigantea.
Majestica.

Maria, $37 \mathrm{cts}$.

Narginatd, $37 \mathrm{cts}$.

Multiflora, $37 \mathrm{cts}$

New Globe, $37 \mathrm{cts}$.

Paragon, 37 cts.
Pride of Peckham.

Queen Adelaide, $37 \mathrm{cts}$.

Rictoria.

Racemiflora elegans.

Reflexa, 37 cts.

Rosea alba, 3 i cts.

Salters, $37 \mathrm{cts}$.

Stanwelliana.

Splendens.

Splendissima.

Stylosa eleyans.

Toddiana.

Venus victrix.

Victory supeib, $37 \mathrm{cts}$.

CRANBERRIES.

We can supply these by 100 or 1000 plants.

\section{RHUBARB.}

The finest varieties can be supplied at reduced rates by 100 or 1000 , to form plantations for market.

\section{GREEN-HOUSE PLANTS.}

In addition to the general collection of all the species and variecies that have been anmounced in various Catrlogues, the following can be supplied singly at the rates numed, and with a discount where a large number are ordered.

Abutilon striatum.

Achimenes coccinea.

grandiflora.

hirsuta.

longiflora.

pedunculata.

picta.

rosea.

Acacia armata

affinis.

dealbata.

farnesiana.

glaucescens.

lophantha.

prostrata.

pubes cens.
Alow, numerous species, 50 to

Araucaria imbricata.

Aster argophyllus.

Astrapea Wallichi, $\$ 2$ to .

Azalea gledstanesii.

, 25 other new Chinese

varieties, $5 v \mathrm{cts}$. to

Bignonia picta. . pandora.

Danisteria fulgens.

Banksia furmosa, and other species

Bletia tankervillæi

Brugmansia Knightii.

Brunsfelsia undulata.

Buonapartia inncea.

Buddlea Lindleyana.

Cactus, all the species, $50 \mathrm{cts}$. to
|\$0 50||Calceolaria, Beauty of Fair Isle.| \$0 37

37

25

37

37

1

Cattley's Chinese Guava.

Rugosa.

Tronbadour.

Widnall's Meteor.

75 Campanula grandis.

25 Ceratonia siliqua.

50 Cereus, many varieties, $50 \mathrm{cts}$, to

75 Cestrum diurnum

75 Cineraria, many varietıes, each

50 Clivea nobilis

50 Coffea arabica.

50 Corræa Cavendishii. :

50 Goodii.: : : 1

75 Harrisi. : : 1

2 Crowea saligna. $\quad \cdot \quad 54$

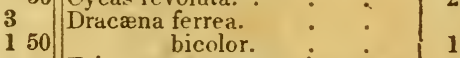

Cycas revoluta. : $:$

1

50

50

5t

Erica, numerous species, $50 \mathrm{cts}$. to 1

150 Erythriua cristagalli, $50 \mathrm{cts}$. to

1 Escallonia rubra.

150 speciosa.

150 Euonymus japonicus, variegatus

Euphorbia elegans.

3 Fabiana imbricata.

Ficus elastica, $50 \mathrm{cts}$

$15 \mathrm{C}$ nitida and others.

1 latifolia, pleno. 
Ciladiolus trimaculata.

For other splendid va-

rieties, see Bulb Catalogue.

Glozinia bicolor.

blanda.

Cartoni.

caulescens.

Colvillii.

gandavencis.

inacrophylla variegata

pudibundus.

rubra.

sup r rba.

tubitlora.

Hæmanthus, varions species.

Ifeliotropium grandiflorum.

Hibiscus rosa sinensi-;, d'ble red

buff and others, $50 \mathrm{cts}$. to double

Hoya carnosa.

Hydrangea Japonica, beautiful

Ilicium foridanum.

Ipomaa Learil.

parvitlcrum. .

Jasminum azoricim, fol var. pubige-um. other pecies, 50 cts to

Kennedia monophrila. rubicunda

Lagerstramia Barclayana.

Lily, new Japan varieties, see Kulb Catalogue.

lisianthus russehranus.

Lobelia ignea.

Lophospermum grandiflorum.

Manettia bicolor.

Marica bicolor, yellow with

black spots, splendid.

Melaleuca, numerous species, 50 cts. to

Melastoma malabathrica. .

Metrosideros, numerous spe-

cies, $50 \mathrm{cts}$. to

Mimosa marginata

Musa, several species, $\$ 1$ to

Mlyrtus tomentosa.

Belgian.

Double flowering.

Italian upright.

Silver striped.

Orange leaved.

Roman. .

Bird nest.

Three leaved, or Jew's. various others.

Yematanthes longipes.

Nepenthes distillatoria.

Nerium album pleno. angustifolium striatum. atropurpureum speciosum candididissimum.
\&0 50"1̄erium curdinalis.

coccinea. pleno.

1

1

75

1 50) giundiflorum. plèno.

1

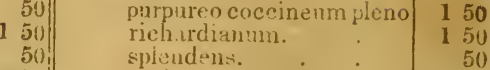

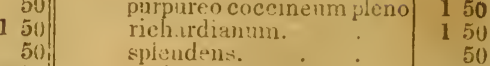

150 variegatum. $\quad: \quad 1$

1

Olea fragrans, 50 cts. to

Oxalis Bowei.

37 Passifiora alato-cerviea jo

50 Passifora ala-ceruleas .

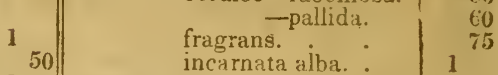

1 Penstemon gentianoide:, cric-

1 cinea.

$50)$ Murrayanim.

50) Pittosporum tobira, fol. var.

1

75

75

Plumbago revolutum.

37 Pulyorala cordifulia. .

37 Pomegranate, Clinese donble

1

variegated.

Desfontaines.

50 Primelea spectabile.

50 Protea argentea. . .

50 Primrose, Chinese double white.

Richardia Ethiopica.

1 Rondeletia speciosa.

Russelia junced.

1

75

Sedum Edwartii, and other

Siphocamylos lantanæfolius.

1

50 Sparaxis grandifiora.

2 Stephonanthus floribundus.

1

E0

50 Swietenia Mahagoni.

50 Tucsonia mollissima.

50

5!!

37
50

Tecoma capensis. pinnatistipula.

51)

51)

75

1

75

5()

50

15

1

1

2

75

50

75

50

1

50

75

1

3

5)

3

1

in

50

5)

$15 i$

150

50

37

50

50

75

Veronica speciosa.

aurantiaca.

Viburnum tinus, 37 cts. to

Violet, Perpetual Tree 